\title{
WSCC 2019: the World Speed Chess Championship
}

Article

Accepted Version

The ICGA WSCC 2019 report

Krabbenbos, J., van den Herik, J. and Haworth, G. (2020) WSCC 2019: the World Speed Chess Championship. ICGA Journal, 1 (4). pp. 237-240. ISSN 1389-6911 doi: https://doi.org/10.3233/ICG-190127 Available at https://centaur.reading.ac.uk/85847/

It is advisable to refer to the publisher's version if you intend to cite from the work. See Guidance on citing.

Published version at: https://content.iospress.com/articles/icga-journal/icg190127

To link to this article DOI: http://dx.doi.org/10.3233/ICG-190127

Publisher: The International Computer Games Association

All outputs in CentAUR are protected by Intellectual Property Rights law, including copyright law. Copyright and IPR is retained by the creators or other copyright holders. Terms and conditions for use of this material are defined in the End User Agreement.

\section{www.reading.ac.uk/centaur}

\section{CentAUR}

Central Archive at the University of Reading

Reading's research outputs online 


\section{WSCC 2019: The World Speed Chess Championship}

Jan Krabbenbos, Jaap van den Herik and Guy Haworth ${ }^{1}$

Amersfoort, the Netherlands, Leiden, the Netherlands and Reading, UK

The ICGA's 2019 'WSCC' World Speed Chess Championship was held alongside its WCCC and WCSC events (Krabbenbos et al., 2019a, 2019b) on the $12^{\text {th }}$ and $15^{\text {th }}$ August at the Venetian Macau Hotel Resort in Macau, China. The location was luxuriously spectacular with every facility, a great place for some of the world's top computer chess authors and operators to meet and celebrate their art and achievements. The ICGA's events were part of the $28^{\text {th }}$ International Joint Conference on Artificial Intelligence (IJCAI-19) which also acted as the main sponsor. The other important sponsor was Digital Game Technology (DGT) who provided the electronic chessboards. Chessbase donated an extra prize for the first position.

All WCCC/WCSC engines other than THE BARON took part as in Table 1. The all-play-all event consisted of ten rounds sequenced for logistical convenience as five double-rounds. Each engine played one opponent with one colour and then the other. The tempo was $5^{\prime}+5^{\prime \prime} / \mathrm{m}$, which makes the operator's experience, accuracy and speed of hand very important.

Table 1

The participants in WSCC 2019 (CPW, 2019)

\begin{tabular}{ccccc}
\hline id & Program & Author(s) & CC & Operator \\
\hline C & CHIRON & Ubaldo Andrea Farina & IT & Ubaldo Andrea Farina \\
G & GINKGO & Frank Schneider & DE & Wolfgang Zugrav \\
J & JONNY & Johannes Zwanzger & DE & Johannes Zwanzger \\
K & KOMODO & Don Dailey, Larry Kaufman \& Mark Lefler & US & Erdogan Günes \\
S & SHREDDER & Stefan Meyer-Kahlen & DE & Stefan Meyer-Kahlen \\
\hline
\end{tabular}

The faster tempo brought its surprises. Game 3, KOMODO lost to JONNY in a dead even position, sadly because of a connection failure with just 15" on the clock. After some 'strange moves', it was then genuinely outplayed in game 16 by CHIRON which, reasonably, was more attracted to an EGT-guaranteed, 28-move mate from move 58 than a 7-man, 16-move mate from move 55.

JONNY took its turn to win all the plaudits - the only unbeaten engine and, even ignoring its fortunate result against KOMODO, the engine with the most wins. Game 2 was a victory over GINKGO as Black, and game 15 was a win over the multiple ICGA champion, SHREDDER.

All games, some lightly annotated and all at GM standard despite the frenetic Blitz tempo, are available for study at human pace from the repository version (Krabbenbos et al., 2019c) of this report. Our heartiest congratulations to Johannes Zwanzger, the 2019 World Speed Chess Champion.

\footnotetext{
${ }^{1}$ Corresponding author: g.haworth@ reading.ac.uk
} 


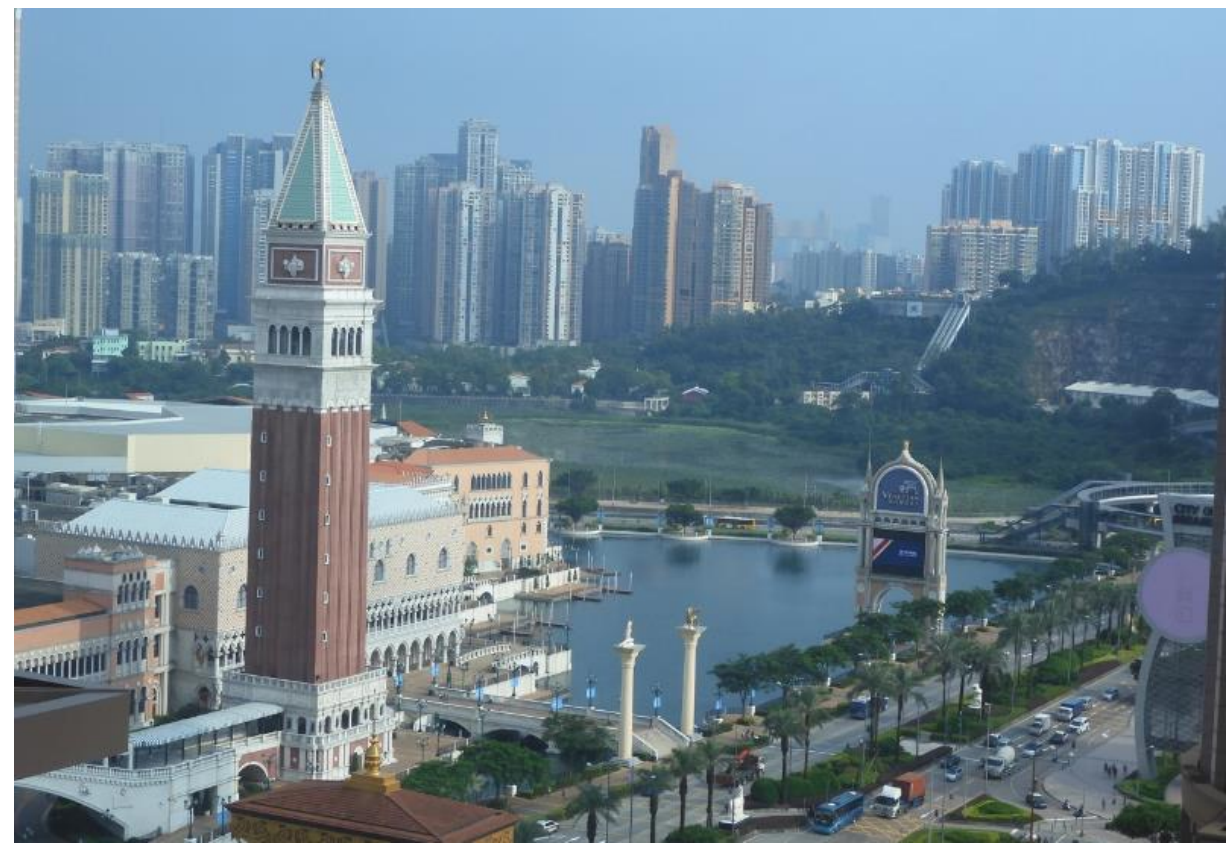

Fig. 1. Part of the Venetian Macau Resort, China.

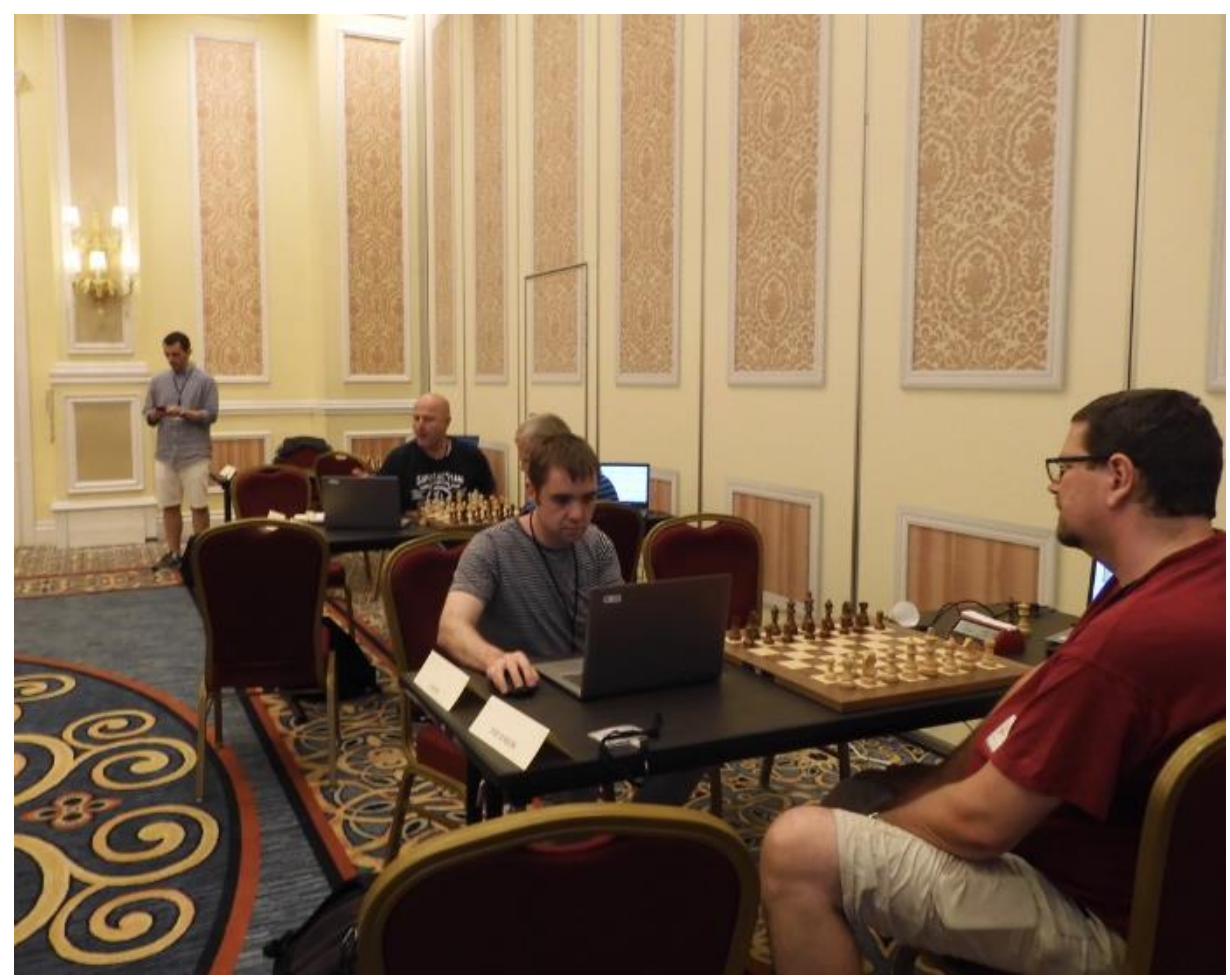

Fig. 2. The ICGA's tournament hall, featuring from right to left,

THE BARON's Richard Pijl, Johannes Zwanzger (JONNY), Stefan Meyer-Kahlen (SHREDDER), Wolfgang Zugrav (GINKGO) and CHIRON's Ubaldo Andrea Farina. 
Table 2

The WSCC 2019 games. $^{2}$

\begin{tabular}{|c|c|c|c|c|c|c|c|c|c|c|c|}
\hline \# & $\mathbf{r}$ & $\mathbf{g}$ & Wh. & Bl. & ECO & Opening & $\# \mathbf{m}$ & Res. & FGen & $7 \mathrm{~m}$ & $6 m$ \\
\hline 01 & 01 & 1 & $\mathrm{~K}$ & $\mathrm{~S}$ & B85 & $\begin{array}{c}\text { Sicilian, Scheveningen, Classical Variation } \\
\text { with ...Qc7 and ...Nc6 }\end{array}$ & 51 & $1 / 2-1 / 2$ & - & & - \\
\hline 02 & 01 & 2 & G & $\mathrm{J}$ & D12 & Queen's Gambit Declined Slav & 58 & $0-1$ & - & & - \\
\hline 03 & 02 & 1 & $\mathrm{~J}$ & $\mathrm{~K}$ & A29 & $\begin{array}{l}\text { English Opening, Four Knights, Kingside Fianchetto: } \\
\text { 1. ... e5 2.Nc3 Nc6 3.Nf3 Nf6 4.g3 }\end{array}$ & 53 & $1-0$ & - & & - \\
\hline 04 & 02 & 2 & $\mathrm{C}$ & $\mathrm{G}$ & B90 & Sicilian, Najdorf & 93 & $1 / 2-1 / 2$ & - & & - \\
\hline 05 & 03 & 1 & $\mathrm{~S}$ & $\mathrm{~J}$ & D10 & Queen's Gambit Declined Slav & 58 & $1 / 2-1 / 2$ & $52 b$ & & - \\
\hline 06 & 03 & 2 & $\mathrm{~K}$ & $\mathrm{C}$ & A28 & $\begin{array}{l}\text { English Opening, Four Knights Sy stem: } \\
\text { 1...e5 2.Nc3 Nc6 3.Nf3 Nf6 (without 4.g3) }\end{array}$ & 68 & $1-0$ & - & & - \\
\hline 07 & 04 & 1 & $\mathrm{C}$ & $\mathrm{S}$ & B47 & Sicilian, Taimanov (Bastrikov) variation & 79 & $1 / 2-1 / 2$ & $70 \mathrm{~b}$ & & - \\
\hline 08 & 04 & 2 & G & $\mathrm{K}$ & B09 & Pirc, Austrian attack & 37 & $1 / 2-1 / 2$ & - & & - \\
\hline 09 & 05 & 1 & $\mathrm{~S}$ & G & E00 & Queen's Pawn Game (including Neo-Indian Attack, & 37 & $1 / 2-1 / 2$ & - & & - \\
\hline 10 & 05 & 2 & $\mathbf{J}$ & $\mathrm{C}$ & E11 & Bogo-Indian Defence & 48 & $1 / 2-1 / 2$ & $38 w$ & & - \\
\hline 11 & 06 & 1 & $\mathrm{~S}$ & $\mathrm{~K}$ & D46 & Queen's Gambit Declined; Semi-Slav 6.Bd3 & 42 & $1 / 2-1 / 2$ & $41 b$ & & - \\
\hline 12 & 06 & 2 & $\mathbf{J}$ & $\mathrm{G}$ & A29 & English Opening, Four Knights, Kingside Fianchetto: & 112 & $1 / 2-1 / 2$ & - & & - \\
\hline 13 & 07 & 1 & $\mathrm{~K}$ & $\mathrm{~J}$ & B67 & $\begin{array}{c}\text { Sicilian, Richter-Rauzer, Rauzer Attack, } \\
7 \ldots \mathrm{a} 6 \text { defence, } 8 . . \mathrm{Bd} 7\end{array}$ & 139 & $1 / 2-1 / 2$ & - & & - \\
\hline 14 & 07 & 2 & G & $\mathrm{C}$ & E10 & Queen's Pawn Game 3.Nf3 & 76 & $1 / 2-1 / 2$ & - & $73 w, 1 / 2-1 / 2$ & - \\
\hline 15 & 08 & 1 & $\mathrm{~J}$ & $\mathrm{~S}$ & B10 & Caro-Kann Defence & 80 & $1-0$ & $34 b$ & & - \\
\hline 16 & 08 & 2 & $\mathrm{C}$ & $\mathrm{K}$ & B06 & Robatsch (Modern) Defence, including Monkey's Bum & 57 & $1-0$ & - & $54 \mathrm{~b}, 1-0$ & - \\
\hline 17 & 09 & 1 & S & $\mathrm{C}$ & D45 & Queen's Gambit Declined; Semi-Slav 5.e3 & 42 & $1 / 2-1 / 2$ & - & & - \\
\hline 18 & 09 & 2 & $\mathrm{~K}$ & G & E97 & $\begin{array}{l}\text { King's Indian, Orthodox, Aronin-Taimanov Variation } \\
\text { (Yugoslav Attack / Mar del Plata Variation) }\end{array}$ & 77 & $1 / 2-1 / 2$ & - & & - \\
\hline 19 & 10 & 1 & $\mathrm{G}$ & $S$ & B49 & Sicilian, Taimanov Variation & 36 & $1 / 2-1 / 2$ & $32 b$ & & - \\
\hline 20 & 10 & 2 & $\mathrm{C}$ & $\mathrm{J}$ & B69 & $\begin{array}{l}\text { Sicilian, Richter-Rauzer, Rauzer Attack, } \\
\text { 7...a6 defence, 11.Bxf6 }\end{array}$ & 124 & $1 / 2-1 / 2$ & - & & - \\
\hline
\end{tabular}

Table 3

The WSCC 2019 cross-table

\begin{tabular}{|c|c|c|c|c|c|c|c|c|c|c|c|c|}
\hline$\# \#$ & id & Program & $\mathbf{J}$ & $\mathrm{C}$ & $\mathbf{G}$ & $\mathbf{K}$ & $\mathbf{S}$ & $\mathbf{W}$ & D & $\mathbf{L}$ & Score & Perf. \% \\
\hline 1 & $\mathrm{~J}$ & JONNY & & $1 / 21 / 2$ & $1 \frac{1}{2}$ & $1 \frac{1}{2}$ & $1 / 21$ & 3 & 5 & 0 & $51 / 2$ & 68.75 \\
\hline 2 & $\mathrm{C}$ & CHIRON & $1 / 21 / 2$ & & $1 / 21 / 2$ & 01 & $1 / 21 / 2$ & 1 & 6 & 1 & 4 & 50.00 \\
\hline 3 & G & GINKGO & $01 / 2$ & $1 / 2^{1 / 2}$ & & $1 / 21 / 2$ & $1 / 21 / 2$ & 0 & 7 & 1 & $31 / 2$ & 43.75 \\
\hline 4 & K & KOMODO & $01 / 2$ & 10 & $1 / 22^{1 / 2}$ & & $1 / 21 / 2$ & 1 & 5 & 2 & $31 / 2$ & 43.75 \\
\hline 5 & $\mathrm{~S}$ & SHREDDER & $1 / 20$ & $1 / 21 / 2$ & $1 / 21 / 2$ & $1 / 21 / 2$ & & 0 & 7 & 1 & $31 / 2$ & 43.75 \\
\hline
\end{tabular}

Table 4

The WSCC 2019 results, round by round

\begin{tabular}{|c|c|c|c|c|c|c|c|c|c|c|c|c|c|}
\hline \# & id & Pro & r1 & r2 & r3 & r4 & r5 & r6 & r7 & r8 & r9 & r10 & Sco \\
\hline 1 & $\mathrm{~J}$ & & & $\mathrm{Gw}^{1 / 2}$ & Kw1 & $\mathrm{Kb}^{1 / 2}$ & \multicolumn{2}{|c|}{ bye } & $\mathrm{Sb}^{1 / 2}$ & Sw1 & $\mathrm{CW}^{1 / 2}$ & $\mathrm{Cb}^{1 / 2}$ & $51 / 2$ \\
\hline 2 & $\mathrm{C}$ & CHIRON & \multicolumn{2}{|c|}{ bye } & $\mathrm{Gw}^{1 / 2}$ & $\mathrm{~Gb}^{1 / 2}$ & $\mathrm{Sw}^{1 / 2}$ & $\mathrm{Sb}^{1 / 2}$ & $\mathrm{~Kb} 0$ & Kw1 & $\mathrm{Jb}^{1 / 2}$ & $\mathrm{JW}^{1 / 2}$ & 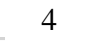 \\
\hline 3 & G & GINKGO & Jw0 & $\mathrm{Jb}^{1 / 2}$ & $\mathrm{Cb}^{1 / 2}$ & $\mathrm{Cw}^{1 / 2}$ & $\mathrm{Kw}^{1 / 2}$ & $\mathrm{~Kb}^{1 / 2}$ & by & ye & $\mathrm{Sb}^{1 / 2}$ & $\mathrm{Sw}^{1 / 2}$ & $31 / 2$ \\
\hline 4 & K & KOMODO & $\mathrm{Sw}^{1 / 2}$ & $\mathrm{Sb}^{1 / 2}$ & $\mathrm{Jb} 0$ & $\mathrm{JW}^{1 / 2}$ & $\mathrm{~Gb}^{1 / 2}$ & $\mathrm{Gw}^{1 / 2}$ & $\mathrm{Cw} 1$ & $\mathrm{Cb} 0$ & by & & $31 / 2$ \\
\hline 5 & $S$ & SHREDDER & $\mathrm{Kb}^{1 / 2}$ & $\mathrm{Kw}^{1 / 2}$ & by & ye & $\mathrm{Cb}^{1 / 2}$ & $\mathrm{Cw}^{1 / 2}$ & $\mathrm{JWW}^{1 / 2}$ & $\mathrm{Jb0}$ & $\mathrm{Gw}^{1 / 2}$ & $\mathrm{~Gb}^{1} 1 / 2$ & $31 / 2$ \\
\hline
\end{tabular}

\footnotetext{
${ }^{2}$ FINALGEN-compatible positions (Müller and Haworth, 2019; Romero, 2012) are noted in column 'FGen'.
} 
Table 5

WCSC 2019 progress, round by round

\begin{tabular}{|c|c|c|c|c|c|c|c|c|c|c|c|}
\hline \# & Program & r1 & $\mathbf{r 2}$ & r3 & r4 & r5 & r6 & r7 & r8 & r9 & r10 \\
\hline 1 & JONNY & 1 & $11 / 2$ & $21 / 2$ & 3 & 3 & 3 & $31 / 2$ & $41 / 2$ & 5 & $51 / 2$ \\
\hline 2 & CHIRON & 0 & 0 & $1 / 2$ & 1 & $11 / 2$ & 2 & 2 & 3 & $31 / 2$ & 4 \\
\hline 3 & GINKGO & 0 & $1 / 2$ & 1 & $1 \frac{1}{2}$ & 2 & $2^{1 / 2}$ & $2 \frac{1}{2}$ & $2 \frac{1}{2}$ & 3 & $31 / 2$ \\
\hline 4 & KOMODO & $1 / 2$ & 1 & 1 & $1 \frac{1}{2}$ & 2 & $21 / 2$ & $31 / 2$ & $31 / 2$ & $31 / 2$ & $31 / 2$ \\
\hline 5 & SHREDDER & $1 / 2$ & 1 & 1 & 1 & $11 / 2$ & 2 & $2^{1 / 2}$ & $2 \frac{1}{2}$ & 3 & $31 / 2$ \\
\hline
\end{tabular}

\section{REFERENCES}

CPW (2019). https://tinyurl.com/icga046. Biographies of programs, authors and operators.

Müller K. and Haworth, G. $M^{\mathrm{c} C}$. (2019). FINALGEN revisited: new discoveries. ICGA Journal, 41(1), 53-60. doi:10.3233/ICG-190095. http://centaur.reading.ac.uk/80432/ provides supporting data, pgn files and an ancillary text with the full analyses and annotations by FINALGEN and the authors.

Krabbenbos, J., van den Herik, H.J. and Haworth, G.McC. (2019a). WCCC 2019: the $25^{\text {th }}$ World Computer Chess Championship. ICGA Journal, 41(4), 206-221. doi: 10.3233/ICG-190125. A lightly annotated pgn file is available from http://centaur.reading.ac.uk/85845/.

Krabbenbos, J., van den Herik, H.J. and Haworth, G.McC. (2019b). WCSC 2019: the $9^{\text {th }}$ World Chess Software Championship. ICGA Journal, 41(4), 222-236. doi: 10.3233/ICG-190126. A lightly annotated pgn file is available from http://centaur.reading.ac.uk/85846/.

Krabbenbos, J., van den Herik, H.J. and Haworth, G.MC. (2019c). WSCC 2019: the 2019 World Speed Computer Chess Championship. ICGA Journal, 41(4), 237-240. doi: 10.3233/ICG-190127. A lightly annotated pgn file is available from http://centaur.reading.ac.u/85847/.

Romero, P.P. (2012) https://tinyurl.com/icga013. FINALGEN: tutorial, download and forum.

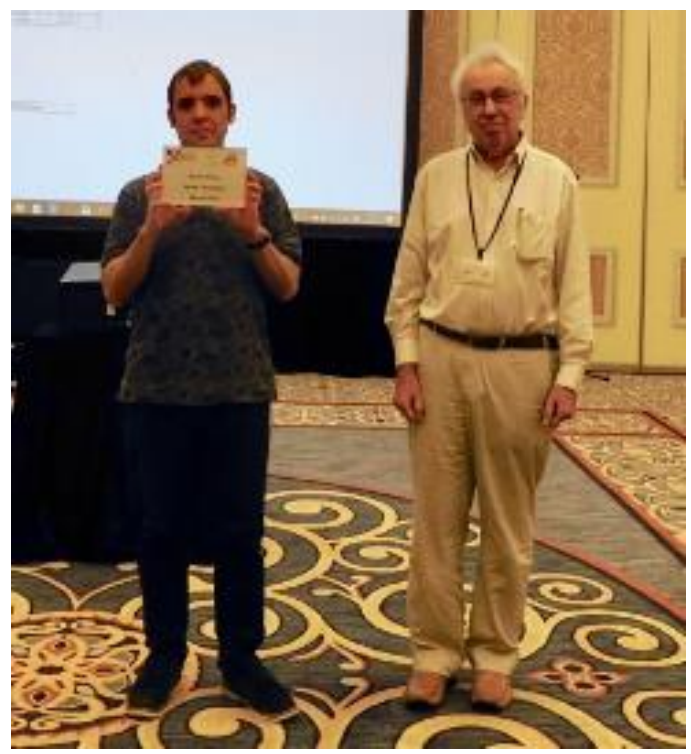

Fig. 3. Johannes Zwanzger, author and operator of JonNY, the 2019 World Speed Chess Champion. David Levy, the long-time and retiring president of the ICGA, presented the prize. 